\title{
Lesion evidence for a critical role of left posterior but not frontal areas in alpha-beta power decreases during context-driven word production
}

\author{
Vitória Piai, ${ }^{1,2}$ (D) Joost Rommers ${ }^{3}$ and Robert T. Knight ${ }^{4}$ \\ ${ }^{1}$ Donders Institute for Brain, Cognition and Behaviour, Donders Centre for Cognition, Radboud University, Montessorilaan 3 , \\ 6525 HR, Nijmegen, the Netherlands \\ 2Department of Medical Psychology, Radboudumc, Nijmegen, the Netherlands \\ ${ }^{3}$ Donders Institute for Brain, Cognition and Behaviour, Donders Centre for Cognitive Neuroimaging, Radboud University, \\ Nijmegen, the Netherlands \\ ${ }^{4}$ Helen Wills Neuroscience Institute and Department of Psychology, University of California Berkeley, Berkeley, CA, USA
}

Keywords: cloze, lexical selection, prediction, priming, spreading activation

\begin{abstract}
Different frequency bands in the electroencephalogram are postulated to support distinct language functions. Studies have suggested that alpha-beta power decreases may index word-retrieval processes. In context-driven word retrieval, participants hear lead-in sentences that either constrain the final word ('He locked the door with the') or not ('She walked in here with the'). The last word is shown as a picture to be named. Previous studies have consistently found alpha-beta power decreases prior to picture onset for constrained relative to unconstrained sentences, localised to the left lateral-temporal and lateral-frontal lobes. However, the relative contribution of temporal versus frontal areas to alpha-beta power decreases is unknown. We recorded the electroencephalogram from patients with stroke lesions encompassing the left lateral-temporal and inferior-parietal regions or left-lateral frontal lobe and from matched controls. Individual participant analyses revealed a behavioural sentence context facilitation effect in all participants, except for in the two patients with extensive lesions to temporal and inferior parietal lobes. We replicated the alpha-beta power decreases prior to picture onset in all participants, except for in the two same patients with extensive posterior lesions. Thus, whereas posterior lesions eliminated the behavioural and oscillatory context effect, frontal lesions did not. Hierarchical clustering analyses of all patients' lesion profiles, and behavioural and electrophysiological effects identified those two patients as having a unique combination of lesion distribution and context effects. These results indicate a critical role for the left lateral-temporal and inferior parietal lobes, but not frontal cortex, in generating the alpha-beta power decreases underlying context-driven word production.
\end{abstract}

\section{Introduction}

Different frequency bands in the electroencephalogram have been postulated to support distinct language functions (e.g. McNab et al., 2012; Mellem et al., 2012; Peelle \& Davis, 2012; Lewis et al., 2015; Piai et al., 2015). Alpha- and beta-band power decreases in speech production were initially linked to the motor cortex (Salmelin et al., 2000; Salmelin \& Sams, 2002), in line with the motor

*Correspondence: Vitória Piai, as above.

E-mail: v.piai.research@gmail.com

Received 14 June 2017, revised 30 August 2017, accepted 30 August 2017

Edited by Ali Mazaheri

Reviewed by: Tony Shahin, University of California, Davis, USA; Katrien Segaert, University of Birmingham, UK

The associated peer review process communications can be found in the online version of this article. literature, which has shown a clear relationship between motor preparation and execution and alpha-beta power decreases (e.g. McFarland et al., 2000; Cheyne, 2013). However, other studies have suggested that alpha-beta oscillations may also index word-retrieval processes, not only in language production but also in language comprehension (Mellem et al., 2012; Piai et al., 2014, 2015; Strauß et al., 2014).

Piai et al. $(2014,2015,2017)$ employed a picture-naming task in which the amount of semantic information provided by a preceding sentence was manipulated. Participants named pictures following sentences with a constrained context, such as 'He locked the door with the [picture: key]', or context-neutral sentences, such as 'She walked in here with the [picture: key]'. An example of the trial structure is shown in Fig. 1A. Picture-naming response times (RTs) are consistently shorter for constrained relative to neutral contexts (Griffin \& Bock, 1998; Piai et al., 2014, 2015, 2017). Left-lateralised alpha-beta power decreases for constrained relative to neutral 

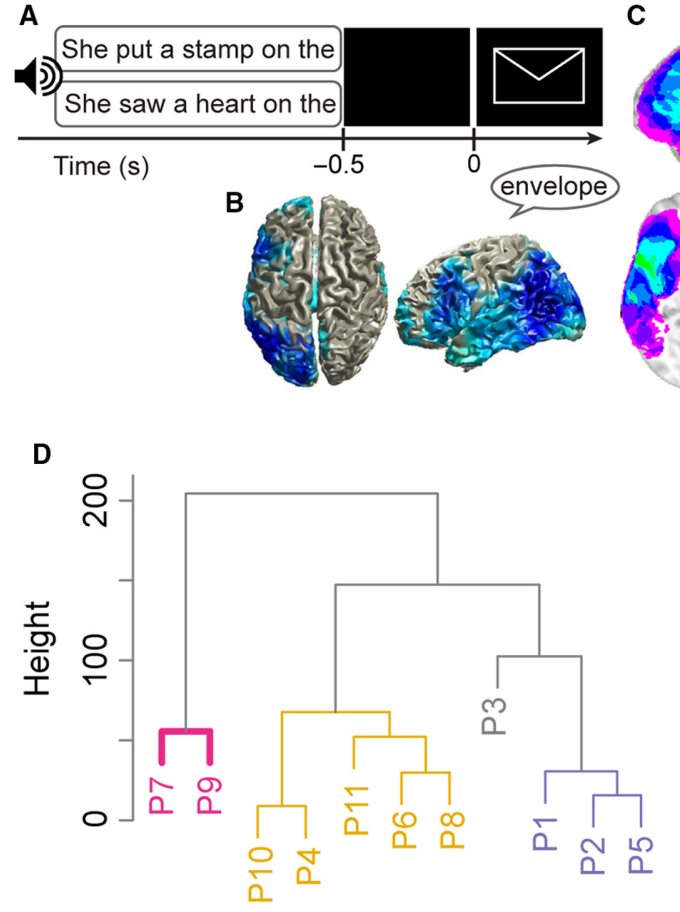
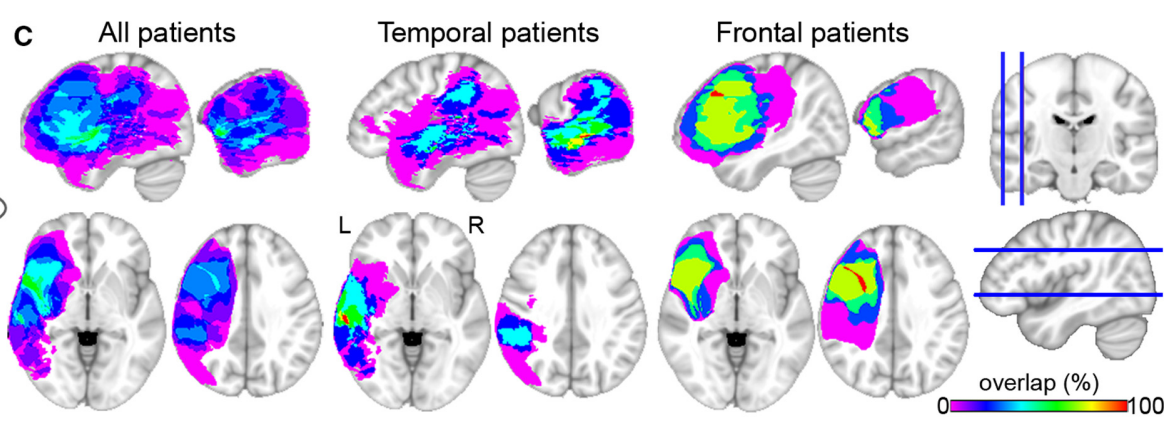

E
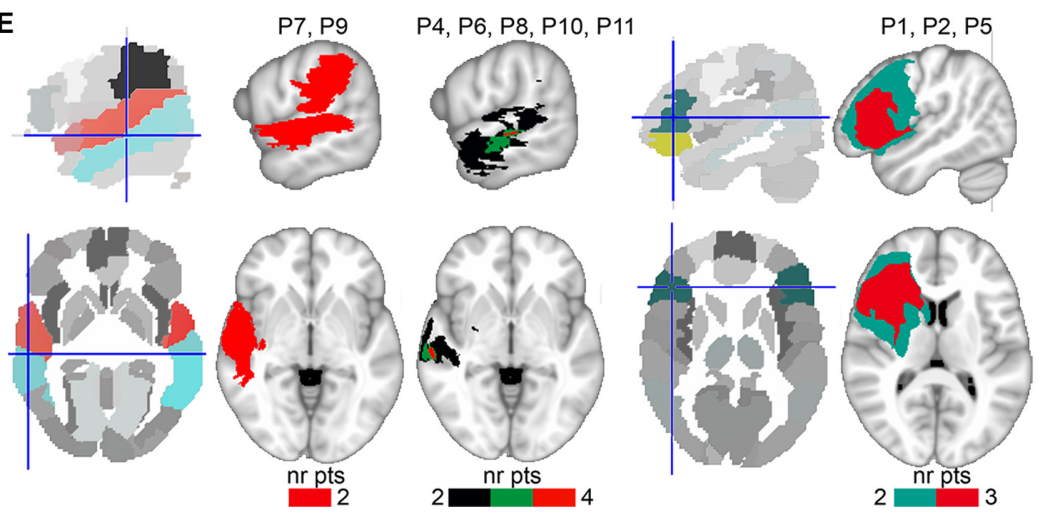

FIG. 1. (A) An example of a trial with constrained (upper) and neutral (lower) sentence contexts with auditory sentences and visual pictures. Only one sentence was presented per trial. (B) Source localisation of the alpha-beta context effect of Piai et al. (2015). (C) Lesion overlap map of all patients (left, $N=11$ ), patients with predominantly temporal lobe lesions (middle, $N=6$ ) and patients with predominantly frontal lobe lesions (right, $N=5$ ). The colour scale indicates the amount of overlap in lesion location, with magenta indicating that only one patient has a lesion in that particular region (i.e. $0 \%$ overlap) and red indicating that all patients have a lesion in that location (i.e. 100\% overlap). (D) Dendrogram of the lesion clusters over left superior temporal, middle temporal, angular, supramarginal and inferior frontal gyri. Significant clusters are indicated by colours. (E). Parcellated brains, with relevant regions of interest in colour, and lesion overlap of patients in each cluster (from D), thresholded at where lesions overlap in at least two patients (nr pts = number of patients). Crosshairs indicate left middle temporal gyrus (left) and left inferior frontal gyrus (right).

contexts have been consistently found after sentence offset but before picture presentation, in healthy young and older adults (Piai et al., 2014, 2015, 2017). These alpha-beta power decreases have been localised to left angular and supramarginal gyri, left anterior and posterior temporal cortex and left inferior frontal gyrus (LIFG), as shown in Fig. 1B (Piai et al., 2015).

Given that the scalp-recorded alpha-beta power decreases index a widespread network of sources, it seems unlikely that these power decreases support a single unitary operation. In particular, it is unclear whether some areas are more critical than others for the alpha-beta power decreases to be measurable over the scalp and for behavioural facilitation to occur in the picture-naming RTs.

Following previous evidence on the roles of the inferior-parietal cortex in conceptual processing (Binder et al., 2009) and of the left temporal lobe in lexical retrieval (Indefrey \& Levelt, 2004; Baldo et al., 2013), the alpha-beta power decreases in those areas could reflect conceptual- and lexical-retrieval processes. Evidence for a causal link between alpha-beta power decreases in the left temporal and inferior-parietal lobes and context-driven word production was obtained by Piai et al. (2017). Six patients with left temporal lesions, in some cases also including the inferior-parietal lobe, performed the context-driven picture-naming task. Behavioural facilitation in the picture-naming RTs as well as the alpha-beta power decreases was replicated in four patients. By contrast, two patients with large lesions, encompassing temporal and inferior-parietal regions, showed no behavioural facilitation and no alpha-beta power decreases.

These findings help further specify the roles of the left temporal and inferior-parietal lobes to which the sources of the alpha-beta context effect had been localised (Piai et al., 2017), but evidence is lacking on the role of the LIFG in this effect. Using eye tracking, lesion-symptom examinations have suggested a critical role for the LIFG in the integration of information with the ongoing sentence context (Nozari et al., 2016). However, the relative contribution of left-temporal versus left-frontal areas to alpha-beta power decreases in word retrieval is unknown.

In this study, we re-analysed the data of the six patients with lesions to the left temporal cortex (Piai et al., 2017) together with additional data of five patients with lesions to the left-frontal cortex (Fig. 1C) and 10 matched controls. For the controls, we expected to replicate the behavioural context facilitation effect and the alpha-beta power decreases before picture onset (Piai et al., 2014, 2015, 2017). Regarding the patients with leftfrontal lesions, based on previous literature indicating the role of the lateral PFC in the use of contextual information to guide behaviour (Fogelson et al., 2009; Nozari et al., 2016), we predicted a diminished context effect in the patients whose lesions encompass the lateral prefrontal cortex, and the LIFG in particular.

\section{Experimental procedures}

The study protocol was approved by the University of California, Berkeley Committee for Protection of Human Subjects, following the declaration of Helsinki. All participants gave written informed consent after the nature of the study was explained and received monetary compensation for their participation. 


\section{Participants}

Eleven patients with stroke-induced lesions to the left lateral-temporal or lateral-frontal cortex participated (five females; median age $=66$, mean $=64, \mathrm{SD}=9$, range $=50-74$; mean years of education =17). The distribution of their lesions is shown in Fig. 1C. Six patients had lesions predominantly in the left-temporal lobe and five in the left-frontal lobe. One additional patient with Wernicke's aphasia and a left-temporal lobe lesion was tested. However, almost half $(48 \%)$ of his responses were errors, so we could not reliably analyse his data. Patients' language abilities from the Western Aphasia Battery (WAB, Kertesz, 1982) were available for nine patients. Four patients had language abilities within normal limits, according to the WAB. Five patients were classified as anomic, characterised by normal auditory verbal comprehension and repetition, but a relatively impaired word-finding ability when speaking. One patient was classified as having conduction aphasia, characterised by normal auditory verbal comprehension, but relatively impaired repetition and word-finding abilities. The two groups of patients did not differ in aphasia severity nor in lesion volume $(t \mathrm{~s}<1, p \mathrm{~s}>0.379)$. All patients were tested at least 12-months post-stroke and were premorbidly right handed. Information on the patients' lesions and language ability is shown in Tables 1 and 2 .

Additionally, ten right-handed controls participated, matched for gender, age and years of education, within \pm 4 years of age and \pm 2 years of education to their matched patient (four females; median and median age $=63.5, \mathrm{SD}=8, \quad$ range $=50-74, \quad t<1$, $P=0.838$; mean years of education $=17, t<1, P>0.659$ ). None of the patients or control participants had a history of psychiatric disturbances, substance abuse, medical complications, multiple neurological events or dementia. All participants were native speakers of American English.

\section{Materials}

The materials were the same used by Piai et al. (2016, 2017). Here, we briefly describe the materials but refer the reader to those reports for further detail. Fifty-one coloured drawings were selected together with their basic-level name. Each item was paired with two sentences for which the target item completed the sentence. All 102 sentences had six syllables. The sentences belonged to two different conditions. In the neutral condition, no specific word was predictable as the final word of the sentence, whereas for the constrained condition, the target word was highly predictable. For each item, the associated sentences had the same two last words. A pretest confirmed differences in the degree of expectancy for the final

TABLE 1. Individual lesion volume and per cent damage to the left middle temporal gyrus (MTG), superior temporal gyrus (STG), angular gyrus (AG), supramarginal gyrus (SMG) and left inferior frontal gyrus (LIFG)

\begin{tabular}{lcccccc}
\hline Patient & Lesion volume & MTG & STG & AG & SMG & LIFG \\
\hline P1 & 52.1 & 0 & 12.9 & 0 & 0 & 59 \\
P2 & 131.76 & 0.1 & 13.1 & 0 & 0 & 93.01 \\
P3 & 122.3 & 0 & 49.8 & 0.5 & 71.6 & 55.1 \\
P4 & 10.09 & 0 & 0 & 0 & 0 & 4.6 \\
P5 & 103.24 & 0 & 10.1 & 0 & 0 & 77.7 \\
P6 & 18.32 & 23.6 & 34 & 2 & 12.5 & 0 \\
P7 & 93.75 & 50.4 & 87.9 & 2.2 & 91.1 & 0 \\
P8 & 103.17 & 17.6 & 33.7 & 5.9 & 32 & 21.4 \\
P9 & 85.82 & 82.6 & 88.6 & 30 & 55.4 & 0 \\
P10 & 4.51 & 6.7 & 3.2 & 0 & 0 & 0 \\
P11 & 36.95 & 56.3 & 22.3 & 0 & 0 & 0 \\
\hline
\end{tabular}

word as a function of context (constrained mean cloze probability $=83 \%$; neutral mean cloze probability $=4 \%, t(50)=45.9$, $P<0.001)$. The sentences in the neutral condition did not have a high cloze probability for any word, with the cloze probability of the most common completion being $23 \%$ on average. Sentences were presented auditorily.

\section{Procedure}

Stimulus presentation and response recording were controlled by Presentation (Neurobehavioral Systems, Albany, CA, USA). Participants were tested individually in an electrically shielded, sound-attenuated, dimly-lit booth. In the practice session, participants trained naming the pictures without blinking and postponing their blinking until a cue was given (three asterisks on the screen). The same blinking cue was used during the experiment proper. The sentences were presented via loudspeakers. A trial began with a fixation cross, displayed continuously during auditory sentence playback. After $1 \mathrm{~s}$, the sentence was presented. After sentence offset, the fixation cross remained on the screen for another $0.5 \mathrm{~s}$ before the picture was displayed for $2 \mathrm{~s}$. Then, the blinking cue appeared for a variable interval between 1.2 and $1.9 \mathrm{~s}$. An example of an experimental trial is given in Fig. 1A.

\section{EEG acquisition}

EEG was recorded from $64 \mathrm{Ag} / \mathrm{AgCl}$ active scalp electrodes (BioSemi, Amsterdam, the Netherlands) mounted in an elastic cap according to the extended 10-20 system. EEG was sampled at $1024 \mathrm{~Hz}$. The horizontal electrooculogram was recorded from electrodes placed on the left and right temples and the vertical electrooculogram from $\mathrm{Fp} 1$ and the electrode positioned below the left eye. Surface electromyogram was recorded from the orbicularis oris muscle with two electrodes placed on the left upper and right lower corner of the mouth.

\section{Behavioural analysis}

Naming responses were monitored online for errors (i.e. disfluent responses, omissions, or incorrect responses). Statistical analyses of the behavioural data were conducted using $\mathrm{R}$ ( $\mathrm{R}$ Development Core Team, 2014). Errors were analysed with logistic regression with condition as a within-participant variable and group as a betweenparticipant variable at an alpha level of 0.05 (two-tailed). Trials corresponding to errors were subsequently excluded from all RT and EEG analyses. Response times were calculated manually using Praat (Boersma \& Weenink, 2013) before the trials were separated by condition.

Participants' median RTs were computed, and an analysis of variance was run on the RTs, with context condition (constrained vs. neutral) as a within-participant variable and group (controls vs. patients) as a between-participant variable at an alpha level of 0.05 (two-tailed). The context effect was also examined at the individual participant level using independent-samples $t$-test across trials.

\section{Lesion analysis}

Lesions were drawn on patients' structural magnetic resonance images (MRIs) by a trained technician and confirmed by a neurologist (RTK). Lesions masks were then normalised to the MNI template. Per cent damage to different areas was determined based on the Automated Anatomical Labeling template in MRIcroN (Rorden 
TABLE 2. Language testing data from the Western Aphasia Battery (WAB) and time elapsed between stroke date $(\mathrm{MPO}=$ months post onset) and WAB testing, and between stroke date and the present EEG experiment. Naming $=$ WAB Naming and Word Finding score $($ maximum $=10)$. Comprehension $=$ WAB Auditory Verbal Comprehension score $($ maximum $=10)$. Sequential commands $=\mathrm{WAB}$ comprehension subtest $($ maximum $=80)$. Aphasia Quotient $(\mathrm{AQ}$, maximum $=100)$. WNL $=$ within normal limit

\begin{tabular}{|c|c|c|c|c|c|c|c|}
\hline Patient & Aphasia type & AQ & Naming & Comprehension & Sequential commands & MPO at WAB & MPO at EEG \\
\hline P1 & WNL & 99.6 & 10 & 10 & 80 & 148 & 174 \\
\hline P2 & Anomic & 91.6 & 9.2 & 10 & 80 & 67 & 209 \\
\hline P4 & NA* & NA* & NA* & NA* & NA* & NA* & 12 \\
\hline P5 & Anomic & 92.1 & 9.3 & 8.83 & 59.5 & 34 & 165 \\
\hline P6 & NA* & $\mathrm{NA}^{*}$ & NA* & NA* & NA* & NA* & 114 \\
\hline P7 & Conduction & 77.9 & 8.6 & 8.55 & 58 & 16 & 23 \\
\hline P10 & WNL & 99.6 & 9.8 & 10 & 80 & 104 & 121 \\
\hline P11 & WNL & 94 & 8.6 & 10 & 80 & 222 & 230 \\
\hline
\end{tabular}

*P4 and P6 were not assessed on the WAB. P6 continued teaching in academia after the stroke. Thus, we are confident that this patient would have been classified as within normal limits by the WAB. P4 conversed without difficulty but complained of word-finding problems.

et al., 2007). To investigate the patients' lesion profile, we used hierarchical clustering over the percentage of damage of the left middle temporal gyrus (MTG), superior temporal gyrus (STG), angular gyrus (AG), supramarginal gyrus (SMG) and LIFG (all entered as separate variables). Cluster analysis is a form of unsupervised learning, used to find structure in the data. It is employed to group elements in so-called clusters such that elements in one same cluster are more similar to each other than to elements in other clusters. The Euclidean distance was used as the distance measure between pairs of observations, and the Ward's criterion was used as the linkage criterion. To validate the cluster solution, multiscale bootstrap resampling was employed with 5000 bootstraps (Suzuki \& Shimodaira, 2006). $P$ values, indicating how well the clusters are supported by the data, were derived from the Approximately Unbiased $P$ value (Suzuki \& Shimodaira, 2006) and only clusters below an alpha level of 0.05 are reported.

\section{EEG analysis}

The analyses were performed using FieldTrip version 20160619 (Oostenveld et al., 2011) in MATLABR2014a. Each electrode was re-referenced offline to averaged mastoids. The data were high-pass filtered at $0.16 \mathrm{~Hz}$ (FieldTrip default filter settings) and segmented into epochs time locked to picture presentation, from $800 \mathrm{~ms}$ pre-picture onset to $300 \mathrm{~ms}$ post-picture onset. All epochs were inspected individually for artefacts such as eye movements, blinks, muscle activity and electrode drifting. For two participants (P6 and C14), rejecting trials with eye blinks resulted in substantial data loss. Therefore, for these two participants, independent component analysis was used to correct for blinks (Jung et al., 2000; as implemented in FieldTrip). Eight peripheral channels (T7, T8, TP7, TP8, F7, F8, FT7, FT8) were excessively noisy (variance $>4$ millivolt) in the majority of participants and were removed from analyses. On average, error- and artefact-free trials comprised 46 trials per condition for controls (no difference in trial numbers between conditions, $t$ (9) $<1, P=0.435$ ), and 44 trials for patients (no difference in trial numbers between conditions, $t(10)<1, P=0.910)$. The number of available trials for patients was on average only two fewer than for controls, $t(19)=-1.795, P=0.089$. Time-resolved spectra were calculated with a modified spectrogram approach, at frequencies ranging from 8 to $25 \mathrm{~Hz}$ (following findings from Piai et al., 2014, $2015,2017)$, with an adaptive sliding time window of three cycles' length (e.g. the window was $300 \mathrm{~ms}$ long at $10 \mathrm{~Hz}$ ). This window was advanced in steps of $10 \mathrm{~ms}$ in the time dimension and in steps of $1 \mathrm{~Hz}$ in the frequency dimension. The data in each window were multiplied with a Hanning taper, and the Fourier transform was taken from the tapered signal. Note that no baseline correction was used.

\section{Statistical analysis}

For each group (patients and controls), we compared the time-frequency representations between the two conditions using a non-parametric cluster-based permutation test (Maris \& Oostenveld, 2007). The test was performed on all available channels, time points (i.e. $-800 \mathrm{~ms}$ to $300 \mathrm{~ms}$ relative to picture onset) and frequencies (i.e. 8-25 Hz). This three-dimensional space was first scanned for time points, frequencies and channels that exhibited a similar difference between the two conditions across participants based on a two-tailed dependent-samples $t$-tests at an alpha level of 0.05 . Time points, frequencies and channels whose $P$ values are lower than the alpha level are selected and clustered on the basis of adjacency. For each cluster, a cluster-level statistic was then calculated by taking the sum of the $t$-values within that cluster. The clusters' statistical significance was then calculated with a Monte Carlo method, for which a permutation distribution is created by randomly partitioning the data into two conditions. Then, the same scanning and clustering procedure are performed for each random partition, and the cluster with the largest summed $t$-values is selected to enter the permutation distribution. This procedure is repeated 1000 times. All cluster-level statistics from the observed data are then compared to this permutation distribution and the proportion of random partitions that yielded a larger cluster test-statistic than that of the observed cluster represents the Monte Carlo estimate of the $P$ value. Using a critical alpha level of 0.05 , we conclude that the constrained and neutral conditions differ from each other significantly if this Monte Carlo $P$-value is smaller than 0.05 .

We also assessed group differences by comparing the relative power differences between the two conditions (i.e. power differences divided by the averaged power across conditions) as a function of group. The same cluster-based permutation procedure was adopted, with the difference that independent-samples $t$-tests were used.

\section{Clustering of variables}

A hierarchical clustering approach was used to divide patients into groups as a function of the anatomical and functional variables 
available. These variables were the following: (i) the percentage of damage of the left MTG, left STG, left AG, left SMG and LIFG (as explained above), (ii) lesion volume, (iii) the behavioural context effect (the mean difference between the two conditions) and (iv) the EEG context effect, that is, the relative power differences between the two conditions averaged over all available channels in the 8$25 \mathrm{~Hz}$ range between $-300 \mathrm{~ms}$ and picture onset (i.e. the significant cluster on the group level, see Results below). The same procedure for the clustering analysis was used as reported above ('Lesion analysis'), except that clusters were evaluated at an alpha level of 0.0125 .

\section{Results}

\section{Lesion profile}

Figure 1D shows how patients are grouped as a function of their lesion profile. The $y$-axis indicates how dissimilar, according to the Euclidean distance, the individual data points and clusters are from each other. Significant clusters are indicated by colours. As can be seen in Fig. 1D, patients P7 and P9 are grouped together and separately from the other patients. This means that the lesion profile of these two patients is more similar to each other than to the lesion profile of the patients in other clusters. The same is the case for the other clusters. The lesion overlap of the patients in each cluster is shown in Fig. 1E thresholded at where lesions overlap in at least two patients. Patients P7 and P9 have a lesion in STG, MTG and SMG. Patients P4, P6, P8, P10 and P11 form a second cluster, with no $100 \%$ lesion overlap among them, but with four patients having an overlapping lesion in MTG. Finally, patients P1, P2 and P5 form a third significant cluster, with all three patients in this cluster having a predominantly LIFG (and insular) lesion.

\section{Sentence context facilitates picture naming}

Table 3 shows the error rates for each patient per condition. Control participants made fewer errors than patients (group level: $0.78 \%$ vs. $5 \%$ respectively, $\beta=-2.387, \quad \mathrm{SE}=0.749, \quad z$-value $=-3.226$, $P<0.002)$. The remaining comparisons were not statistically significant, all $p \mathrm{~s}>0.176$.

Figure 2 shows the participant-level median RTs per condition (left panel) and the individual magnitude and significance of the context effect, with $95 \%$ confidence intervals (right panel). On the group level, responses in the constrained condition were faster than in the neutral condition $\left(F_{1,19}=101, P<0.001\right)$, and patients were overall slower than controls $\left(F_{1,19}=5.05, P=0.037\right)$, but the interaction was not significant $\left(F_{1,19}<1\right)$. On the individual level, all control participants and all but two patients (P7 and P9) were reliably faster on constrained than neutral trials, indicating a robust within-participant behavioural facilitation effect.

\section{Sentence context modulates pre-picture alpha-beta power}

In both controls and patients, we observed about 15-20\% power decreases (relative to the average across conditions) in the alphabeta range prior to picture presentation, as shown in Fig. 3A. Cluster-based permutation tests indicated a statistically significant context effect for both controls (Monte Carlo $P=0.039$ ) and patients (Monte Carlo $P<0.001$ ) that could be attributed to a spatio-spectro-temporal cluster of power decreases in each group. These clusters were detected between 8 and $25 \mathrm{~Hz}$ and -0.3 to $0 \mathrm{~s}$ in both groups, in the channels indicated in white in the topographies. The interaction between context condition and group did not yield any significant clusters (both when all patients were included and when patients P7 and P9 were excluded). This result is to be expected given that, on the group level, both groups show a significant context effect associated with a cluster in the $8-25 \mathrm{~Hz}$ range prior to picture onset.

Individual EEG effects are shown in Fig. 3B. Patients P7 and P9 showed the weakest alpha-beta power decreases over the scalp. EEG effects averaged over clusters of patients based on their lesion profiles (Fig. 1D) are shown in Fig. 3C. These topographies indicate that for the patients with lesions overlapping in the mid portion of the MTG (P4, P6, P8, P10, P11, the yellow cluster in Fig. 1D), the scalp effect is bilateral, with a slight right-lateralised bias. For the patients with a predominantly LIFG lesion (P1, P2, P5, the purple cluster in Fig. 1D), the scalp effect is largely left-lateralised. The average over P7 and P9 (the pink cluster in Fig. 1D) confirms that these two patients have the weakest EEG effects.

\section{Extensive posterior lesions and weak behavioural and oscillatory effects co-occur}

Figure 4 shows the results of the hierarchical clustering analyses. Significant clusters are indicated by filled lines. When all functional and anatomical variables were entered, the clustering procedure

TABLE 3. Error rate (in percentage) and error type per patient and condition. $\mathrm{C}=$ constrained; $\mathrm{N}=$ neutral

\begin{tabular}{|c|c|c|c|c|}
\hline Patient & $\% \mathrm{C}$ & $\% \mathrm{~N}$ & Type: constrained & Type: neutral \\
\hline $\mathrm{P} 1$ & 3.9 & 2.0 & Hesitation (1), no response (1) & Did not identify picture (1) \\
\hline $\mathrm{P} 2$ & 7.8 & 11.8 & No response (1), hesitation with correct response (3) & Unclassifiable (1), no response (1), hesitation with correct response (4) \\
\hline P3 & 3.9 & 9.8 & Unclassifiable (1), semantically related response (1) & $\begin{array}{l}\text { No response (1), did not identify picture (1), unclassifiable (1), } \\
\text { semantically related response (2) }\end{array}$ \\
\hline P4 & 2.0 & 0.0 & Hesitation with correct response (1) & - \\
\hline P5 & 5.9 & 0.0 & No response (3) & - \\
\hline P6 & 0.0 & 2.0 & - & No response \\
\hline P7 & 5.9 & 9.8 & No response (3), phonological paraphasia (1) & $\begin{array}{l}\text { No response (3), semantically related response (1), did not } \\
\text { identify picture (1) }\end{array}$ \\
\hline P8 & 2.0 & 9.8 & No response $(1)$ & $\begin{array}{l}\text { No response (3), hesitation with incorrect response (1), hesitation } \\
\text { with correct response (1) }\end{array}$ \\
\hline P9 & 5.9 & 7.8 & Hesitation with correct response (3) & Hesitation with correct response (4) \\
\hline P10 & 0.0 & 2.0 & - & Semantically related response (1) \\
\hline P11 & 7.8 & 9.8 & $\begin{array}{l}\text { Did not identify picture (1), hesitation } \\
\text { with correct response (2), hesitation } \\
\text { with incorrect response (1) }\end{array}$ & $\begin{array}{l}\text { Did not identify picture }(2) \text {, phonological paraphasia }(1) \text {, } \\
\text { hesitation with correct response }(2)\end{array}$ \\
\hline
\end{tabular}



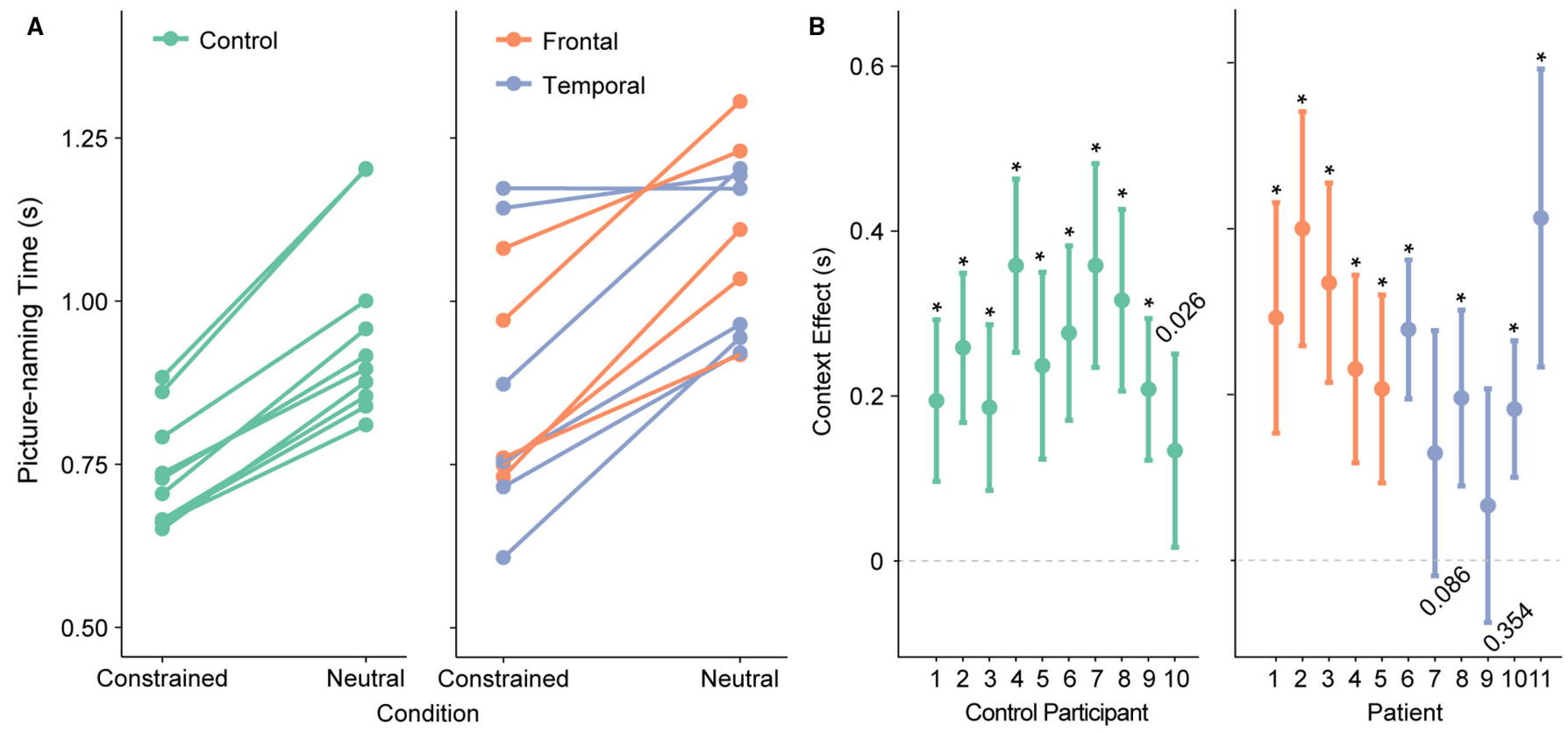

FIG. 2. (A) Median picture-naming times for each participant and condition. (B) Context facilitation effect (neutral-constrained) with $95 \%$ confidence intervals and $P$ value for each participant. Asterisks indicate $P<0.001$.

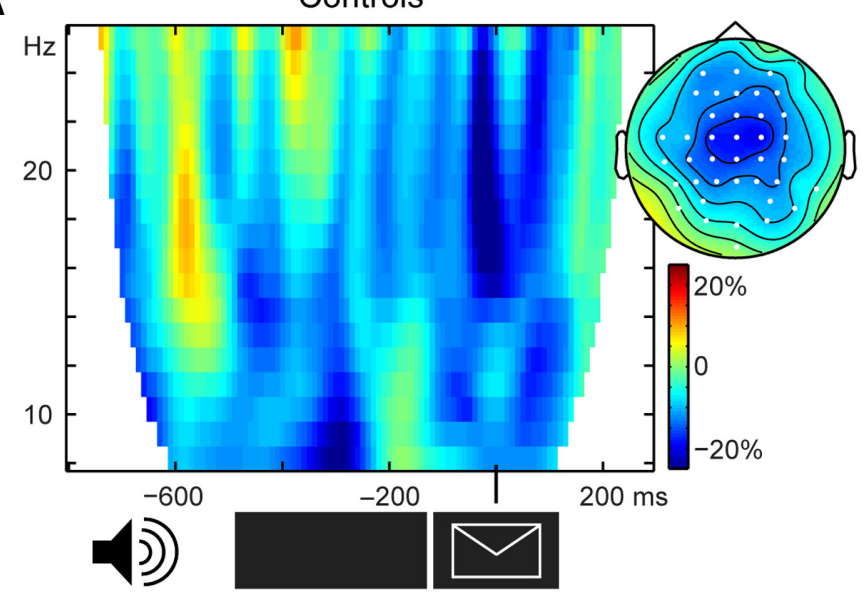

A

Controls

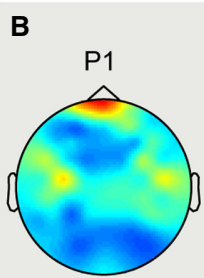

P6

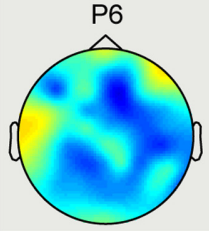

P2

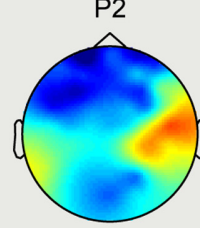

P7

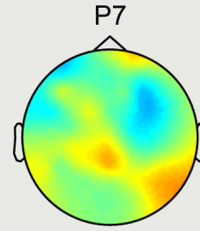

Patients

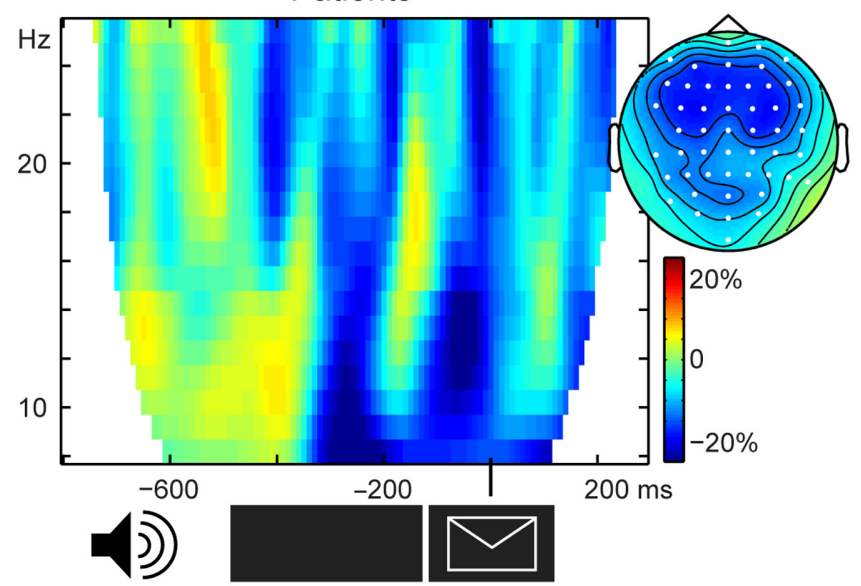

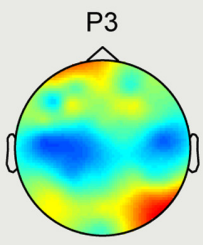

P8

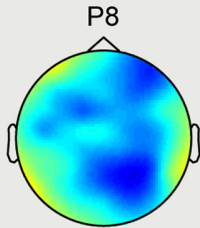

P4

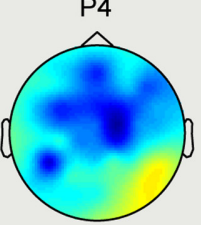

P9

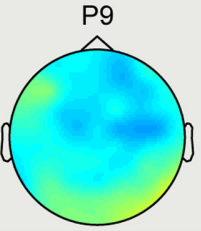

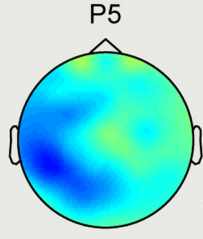

P10

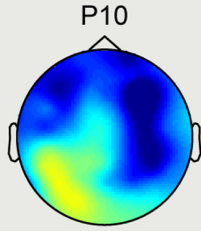

C

$\mathrm{P} 4, \mathrm{P} 6, \mathrm{P} 8, \mathrm{P} 10, \mathrm{P} 11$
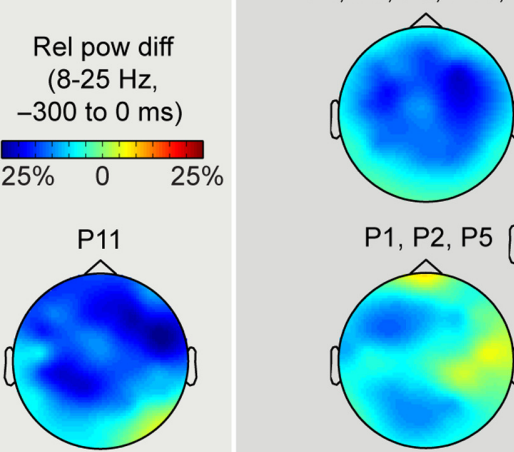

Rel pow diff

$(8-25 \mathrm{~Hz}$,

-300 to $0 \mathrm{~ms}$ )

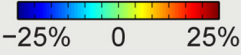

FIG. 3. (A) Time-frequency representations of the context effect (in relative power changes) averaged over the channels associated with the significant clusters, indicated in white on the topographical maps. The topographical distributions of the significant cluster $(8-25 \mathrm{~Hz},-0.3$ to 0 s) are shown for each group. (B) Patients' individual topographical distribution of the context effect $(8-25 \mathrm{~Hz},-0.3$ to $0 \mathrm{~s}$ ), expressed as relative power changes. (C) Topographical distribution of the context effect (in relative power changes) averaged over the patients in each cluster from Figure 1D. 
yielded one distinct significant cluster, with patients P7 and P9 separately from the other patients. We ran additional analyses to further examine the uniqueness of this clustering solution. When clustering was performed on all variables but the EEG (i.e. pre-picture alphabeta power), patients P7 and P9 were again clustered together, separately from the other patients. Performing the clustering procedure on all variables but lesion volume also yielded a unique cluster of patients P7 and P9. Finally, when all variables were entered but the behavioural effect, patients P7 and P9 again clustered together. In summary, despite the changing configuration of the dendrograms with different variables entered in the clustering analysis, patients P7 and P9 had a unique constellation of functional and anatomical variables different from the other patients.

\section{Discussion}

In the present study, patients with stroke-induced lesions to the left temporal, inferior-parietal or frontal lobes and matched controls performed a context-driven picture-naming task while their EEG was recorded.

Replicating previous findings (Griffin \& Bock, 1998; Piai et al., 2014, 2015, 2017), all healthy-control participants showed shorter picture-naming times and alpha-beta power decreases before picture onset following constrained sentences relative to neutral sentences. This pattern of behavioural and electrophysiological effects was also found for all patients, except for two patients with extensive lesions to the temporal and inferior-parietal lobes (P7 and P9). We used hierarchical clustering to group patients as a function of their lesions profiles, and behavioural and oscillatory effects. These analyses indicated that P7 and P9 had a unique combination of lesion distribution and lack of behavioural and electrophysiological context effects. By contrast, no such association was found between the LIFG and alpha-beta power decreases. Together, these results provide evidence for a causal link between posterior alpha-beta power decreases (i.e. in the left lateral-temporal and inferior-parietal lobes) and context-driven word retrieval.

Regarding the naming latencies, the fact that patients with PFC lesions still benefitted from constraining sentence contexts is
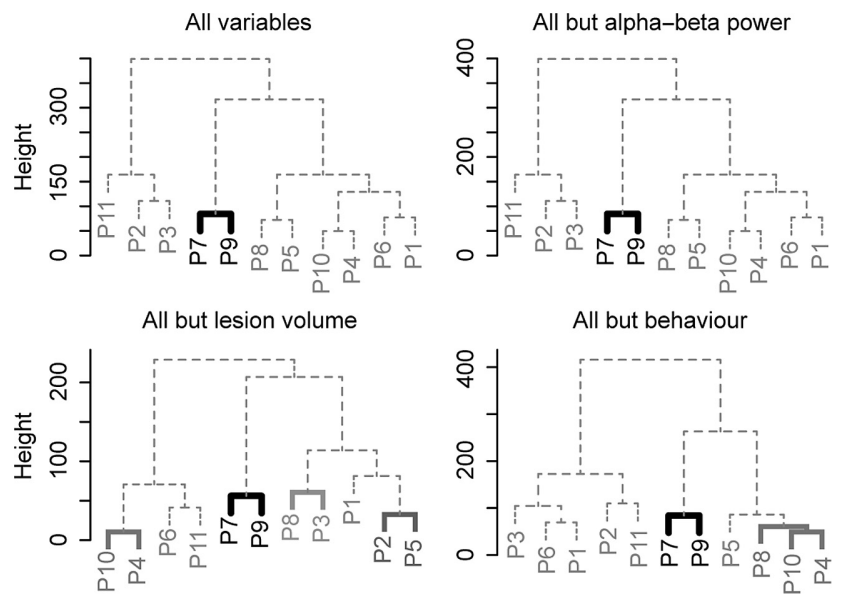

FIG. 4. Dendrograms of the patient clusters for all functional (EEG, behaviour) and anatomical (per cent lesion in regions of interest, lesion volume) variables (top left), all variables but EEG (top right), all variables but lesion volume (bottom left), and all variables but behaviour (bottom right). Significant clusters (at an alpha level of 0.0125 ) are indicated by filled, thicker lines. noteworthy as previous studies have highlighted the critical role of the lateral PFC in people's ability to use contextual information to guide behaviour. Fogelson et al. (2009) examined seven patients with lateral PFC lesions (with the greatest lesion overlap in the dorsolateral PFC) performing a non-verbal target-detection task in which patients responded manually to a target following a predictive or a random sequence. Both behavioural and neurophysiological evidence were obtained for patients' impaired ability to employ the local context (i.e. the sequence) to anticipate the target and respond to it, contrary to our findings. However, the predictive context in their study consisted of abstract visual sequences, whereas in our study, the sequences consisted of meaningful words. This aspect of our materials may have decreased the demands on control and selection processes, supported by the lateral PFC.

A similar account is likely for the differences between our findings and those of Nozari et al. (2016). Using eye tracking, Nozari et al. compared the anticipatory eye movements of four patients with LIFG lesions to those of three patients with lesions to the left temporal and parietal lobes. Patients watched a screen with four pictures (e.g. car, hat, banana, flashlight) while hearing sentences with restrictive or non-restrictive verbs (e.g. 'She will drive the' vs. 'She will study the'). The patients with left posterior lesions looked at the target (here, the pictured car) earlier following a restrictive verb than the patients with LIFG lesions. The authors concluded that LIFG damage impaired patients' ability to use the semantic contextual cues present in the sentence to relate it to the target picture. However, the visual-world paradigm employed by Nozari et al. (2016) requires participants to integrate visual information from the pictures with the auditory sentence and to avoid influences from irrelevant pictures in the display, possibly increasing control and selection demands on the PFC. In our case, spreading activation in the lexico-semantic network through the information in the sentence alone may have been sufficient to guide retrieval of the picture concept and its name.

The present findings begin to illuminate the relative roles of left temporal, inferior-parietal and frontal areas in the alpha-beta context effect in word production. Whereas posterior lesions, including the left temporal and inferior-parietal lobes, eliminated alpha-beta power decreases prior to picture onset, LIFG lesions did not. Thus, out of the various sources implicated in eliciting the context alpha-beta power decreases (Piai et al., 2015), the temporal and inferior-parietal areas might play a more critical role than the left inferior frontal areas. Given the functional roles associated with these brain areas, alpha-beta power decreases could reflect a mix of core semantic memory and lexical-retrieval processes (Binder et al., 2009; Schwartz et al., 2009; Baldo et al., 2013), along with additional frontal control processes that may have been less critical in the task employed in this study.

Previous studies have found correlational evidence for the role of alpha-beta power decreases in lexical retrieval (e.g. Mellem et al., 2012; Brennan et al., 2014; Piai et al., 2015). The present study contributes a causal link between alpha-beta power decreases in the left temporal and inferior-parietal lobes and context-driven word production. However, it is still unclear whether one sole oscillatory signature (i.e. alpha-beta power decreases) indexes lexical retrieval or whether distinct aspects of this process are indexed by different frequency bands (e.g. Bastiaansen et al., 2005; Marinković et al., 2012; Mellem et al., 2012; Piai et al., 2014). Future studies will hopefully clarify this issue.

Understanding language-related alpha-beta oscillations is not only relevant for theory but also for improving clinical applications of these neuronal signatures. Previous studies have used alpha-beta 
power modulations as an index of language function to understand hemispheric functional re-organisation after left-hemisphere strokes (Meltzer et al., 2013; Kielar et al., 2016; Piai et al., 2017) and to determine hemispheric dominance for language preoperatively in neurosurgical patients (Findlay et al., 2012). We suggest that theoretical and clinical progress on this subject will be mutually informative to both the neurobiology of language and patient care.

In conclusion, we obtained behavioural and neurophysiological evidence for a causal link between alpha-beta power decreases in the left lateral-temporal and inferior-parietal lobes and context-driven word production.

\section{Acknowledgements}

The authors are grateful for the patients and their families, as well as for the other volunteer participants for taking part in this study. We would like to thank Donatella Scabini and Brian Curran for patient delineation, Brian Curran, Clay Clayworth and Callum Dewar for lesion reconstruction, Amber Moncrief and Selvi Paulraj for helping design the materials, Paige Mumford and Laura Agee for help with audio recordings, Kristoffer Dahlslätt for invaluable discussions, and the members of the Center for Aphasia and Related Disorders at the VAHCS in Martinez, CA, for neuropsychological testing.

\section{Conflict of interest}

The authors declare no conflict of interest.

\section{Funding}

This work is supported by grants from the Netherlands Organization for Scientific Research (446-13-009 to V.P., 275-89-032 to J.R.), the National Institutes of Health (NINDS R37 NS21135 to R.T.K), and by the Nielsen Corporation.

\section{Data accessibility}

This article's supporting data and materials can be obtained upon request.

\section{References}

Baldo, J.V., Arévalo, A., Patterson, J.P. \& Dronkers, N.F. (2013) Grey and white matter correlates of picture naming: evidence from a voxel-based lesion analysis of the Boston Naming Test. Cortex, 49, 658-667.

Bastiaansen, M.C.M., van der Linden, M., Ter Keurs, M., Dijkstra, T. \& Hagoort, P. (2005) Theta responses are involved in lexical-semantic retrieval during language processing. J. Cogn. Neurosci., 17, 530-541.

Binder, J.R., Desai, R.H., Graves, W.W. \& Conant, L.L. (2009) Where is the semantic system? A critical review and meta-analysis of 120 functional neuroimaging studies. Cereb. Cortex, 19, 2767-2796.

Boersma, P. \& Weenink, D. (2013). Praat: doing phonetics by computer (Version 5.3.42). Available www.praat.org

Brennan, J., Lignos, C., Embick, D. \& Roberts, T.P.L. (2014) Spectro-temporal correlates of lexical access during auditory lexical decision. Brain Lang., 133, 39-46.

Cheyne, D.O. (2013) MEG studies of sensorimotor rhythms: a review. Exp. Neurol., 245, 27-39.

Findlay, A.M., Ambrose, J.B., Cahn-Weiner, D.A., Houde, J.F., Honma, S., Hinkley, L.B.N., Berger, M.S., Nagarajan, S.S., et al. (2012) Dynamics of hemispheric dominance for language assessed by magnetoencephalographic imaging. Ann. Neurol., 71, 668-686.

Fogelson, N., Shah, M., Scabini, D. \& Knight, R.T. (2009) Prefrontal cortex is critical for contextual processing: evidence from brain lesions. Brain, 132, 3002-3010.

Griffin, Z.M. \& Bock, K.J. (1998) Constraint, word frequency, and the relationship between lexical processing levels in spoken word production. $J$. Mem. Lang., 38, 313-338.
Indefrey, P. \& Levelt, W.J.M. (2004) The spatial and temporal signatures of word production components. Cognition, 92, 101-144.

Jung, T.P., Makeig, S., Humphries, C., Lee, T.W., M.J., M., Iragui, V. \& Sejnowski, T.J. (2000) Removing electroencephalographic artifacts by blind source separation. Psychophysiology, 37, 163-178.

Kertesz, A. (1982). Western Aphasia Battery Test Manual. Grune \& Stratton, New York.

Kielar, A., Deschamps, T., Jokel, R. \& Meltzer, J.A. (2016) Functional reorganization of language networks for semantics and syntax in chronic stroke: evidence from MEG. Hum. Brain Mapp., 37, 2869-2893.

Lewis, A.G., Wang, L. \& Bastiaansen, M. (2015) Fast oscillatory dynamics during language comprehension: unification versus maintenance and prediction? Brain Lang., 148, 51-63.

Marinković, K., Rosen, B.Q., Cox, B. \& Kovacevic, S. (2012) Event-related theta power during lexical-semantic retrieval and decision conflict is modulated by alcohol intoxication: anatomically constrained MEG. Front. Psychol., 3, 121.

Maris, E. \& Oostenveld, R. (2007) Nonparametric statistical testing of EEGand MEG-data. J. Neurosci. Meth., 164, 177-190.

McFarland, D.J., Miner, L.A., Vaughan, T.M. \& Wolpaw, J.R. (2000) Mu and beta rhythm topographies during motor imagery and actual movements. Brain Topogr., 12, 177-186.

McNab, F., Hillebrand, A., Swithenby, S.J. \& Rippon, G. (2012) Combining temporal and spectral information with spatial mapping to identify differences between phonological and semantic networks: a magnetoencephalographic approach. Front. Psychol., 3, 273.

Mellem, M.S., Bastiaansen, M.C.M., Pilgrim, L.K., Medvedev, A.V. \& Friedman, R.B. (2012) Word class and context affect alpha-band oscillatory dynamics in an older population. Front. Psychol., 3, 97.

Meltzer, J.A., Wagage, S., Ryder, J., Solomon, B. \& Braun, A.R. (2013) Adaptive significance of right hemisphere activation in aphasic language comprehension. Neuropsychologia, 51, 1248-1259.

Nozari, N., Mirman, D. \& Thompson-Schill, S.L. (2016) The ventrolateral prefrontal cortex facilitates processing of sentential context to locate referents. Brain Lang., 157-158, 1-13.

Oostenveld, R., Fries, P., Maris, E. \& Schoffelen, J.-M. (2011) FieldTrip: open source software for advanced analysis of MEG, EEG, and invasive electrophysiological data. Comput. Intell. Neurosci., 2011, 1-9.

Peelle, J.E. \& Davis, M.H. (2012) Neural oscillations carry speech rhythm through to comprehension. Front. Psychol., 3, 320.

Piai, V., Roelofs, A. \& Maris, E. (2014) Oscillatory brain responses in spoken word production reflect lexical frequency and sentential constraint. Neuropsychologia, 53, 146-156.

Piai, V., Roelofs, A., Rommers, J. \& Maris, E. (2015) Beta oscillations reflect memory and motor aspects of spoken word production. Hum. Brain Mapp., 36, 2767-2780.

Piai, V., Anderson, K.L., Lin, J.J., Dewar, C., Parvizi, J., Dronkers, N.F. \& Knight, R.T. (2016) Direct brain recordings reveal hippocampal rhythm underpinnings of language processing. Proc. Natl. Acad. Sci. USA, 113, 11366-11371.

Piai, V., Meyer, L., Dronkers, N.F. \& Knight, R.T. (2017) Neuroplasticity of language in left-hemisphere stroke: evidence linking subsecond electrophysiology and structural connections. Hum. Brain Mapp., 38, 31513162 .

R Development Core Team. (2014). R: A Language and Environment for Statistical Computing. R Development Core, Vienna, Austria. Available http://www.r-project.org/

Rorden, C., Karnath, H.-O. \& Bonilha, L. (2007) Improving lesion-symptom mapping. J. Cogn. Neurosci., 19, 1081-1088.

Salmelin, R. \& Sams, M. (2002) Motor cortex involvement during verbal versus non-verbal lip and tongue movements. Hum. Brain Mapp., 16, 8191.

Salmelin, R., Schnitzler, A., Schmitz, F. \& Freund, H.J. (2000) Single word reading in developmental stutterers and fluent speakers. Brain, 123, 11841202.

Schwartz, M.F., Kimberg, D.Y., Walker, G.M., Faseyitan, O., Brecher, A., Dell, G.S. \& Coslett, H.B. (2009) Anterior temporal involvement in semantic word retrieval: voxel-based lesion-symptom mapping evidence from aphasia. Brain, 132, 3411-3427.

Strauß, A., Kotz, S.A., Scharinger, M. \& Obleser, J. (2014) Alpha and theta brain oscillations index dissociable processes in spoken word recognition. Neurolmage, 97, 387-395.

Suzuki, R. \& Shimodaira, H. (2006) Pvclust: an R package for assessing the uncertainty in hierarchical clustering. Bioinformatics, 22, 1540-1542. 